\title{
EMBEDDING CATEGORIES WITH EXACTNESS INTO THEIR ABELIANIZATION
}

\author{
MURRAY ADELMAN
}

(Received 10 February 1976; revised 2 March 1976)

\begin{abstract}
We give a partial answer to the following questions: Given an additive category and a class of sequences, under what conditions is the universal functor to the abelian category faithful and what other sequences are taken to exact sequences?

The "answers" to these questions appear as theorems 2.2 and 3.2 respectively and amount to a weakening of the condition that there be enough relative projectives.

We then characterize those additive categories with exactness which have the property that all relative exact sequences are determined by a small set of functors into the category of abelian groups.
\end{abstract}

\section{\$1. Introduction}

An additive category with exactness is defined to be an additive category $\mathscr{A}$ together with a class $\mathscr{E}$ of sequences of the form

$$
E^{\prime} \stackrel{e^{\prime}}{\longrightarrow} E \stackrel{e}{\longrightarrow} E^{\prime \prime}
$$

The structure is denoted by $(\mathscr{A}, \mathscr{E})$ (Freyd 1965). We do not require that $e e^{\prime}=0$, if so the sequence will be called a complex. Since we are dealing only with additive categories, we will assume that $\mathscr{E}$ contains all split exact sequences. An exact functor from $(\mathscr{A}, \mathscr{E})$ to $\left(\mathscr{A}^{\prime}, \mathscr{E}^{\prime}\right)$ is an additive functor $F: A \rightarrow \mathbb{A}^{\prime}$ with the additional condition that if $E^{\prime} \rightarrow E \rightarrow E^{\prime \prime}$ is in $\mathscr{E}$ then $F E^{\prime} \rightarrow F E \rightarrow F E^{\prime \prime}$ is in $\mathscr{E}^{\prime}$.

Let $\mathbf{E}$ denote the category of additive categories with exactness and natural equivalence classes of exact functors. There is a natural inclusion of $\mathbf{A} b$, the category of abelian categories with natural equivalence classes of exact functors, into $\mathbf{E}$. If $\mathscr{A}$ is an abelian category, let $\mathscr{C}$ be the class of all sequences with zero homology. Homology for sequences that are not complexes are defined in Faber $(1965)$. $(\mathscr{A}, \mathscr{E})$ constructed this way is 
obviously an additive category with exactness. The inclusion of $\mathbf{A} b$ into $\mathbf{E}$ is full.

The left adjoint to the above inclusion is constructed in Adelman (1973). We denote the image of $(\mathscr{A}, \mathscr{E})$ under the left adjoint by $\mathscr{E}(\mathscr{A})$ and the unit of the adjunction by $J_{\mathscr{A}}$. We give necessary conditions that $J_{\mathscr{A}}$ be an embedding, or equivalently, that $(\mathscr{A}, \mathscr{E})$ can be exactly embedded into an abelian category.

Let $(\mathscr{A}, \mathscr{E})$ be a category with exactness. We will denote by $\overline{\mathscr{E}}$ the exact saturation of $\mathscr{E}$. That is, $\overline{\mathscr{E}}$ is the class of all sequences sent to zero homology sequences by all exact functors with domain $\mathscr{A}$ and abelian codomain. We give necessary conditions that $\overline{\mathscr{E}}$ be determined by the projectives relative to $\mathscr{E}$.

Finally, we give necessary and sufficient conditions on an additive category $\mathscr{A}$ that guarantee that, for any $\mathscr{E}, \overline{\mathscr{E}}$ will be determined by a small set of functors into the category of abelian groups. The conditions look like Freyd's one-body condition, but are in fact stronger. See Freyd (1973).

The category of abelian groups will be denoted by $\mathscr{G}$.

\section{Embedding of categories with exactness}

LemMA 2.1. Let $\mathscr{A}$ be an abelian category and $\mathscr{S}$ a Serre class in $\mathscr{A}$ (Adelman (1973) or Gabriel (1967) "Thick Subcategories"). Let $\mathcal{N} \mathscr{S}$ denote the abelian category obtained by inverting all maps whose kernel and cokernel are in $\mathscr{P}$. Suppose $K: \mathscr{A} \rightarrow \mathscr{A} / \mathscr{S}$ is the canonical exact functor. Then, for any $f: A \rightarrow B$ in $\mathscr{A}, K(f)=0$ if and only if there is a factoring $A \rightarrow S \rightarrow B=f$ in with $S$ in $\mathscr{S}$.

Proof. If $K(f)=0$ then there is a diagram of the form

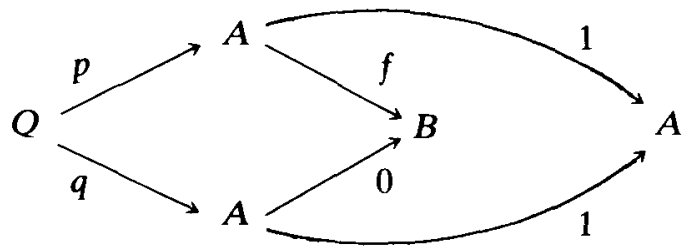

in $\mathscr{A}$ where the inner and outer "diamonds" commute, and with the kernel and cokernel of $p$ and $q$ in $\mathscr{S}$. We obtain $p=q$ and $f p=0$. Let $S=\operatorname{cok} p$.

THEOREM 2.2. Let $(\mathscr{A}, \mathscr{E})$ be an additive category with exactness with the property that for every $A$ in $\mathscr{A}$ there is an abelian category $\mathscr{B}$ and a family of exact functors $\left\{F_{A}^{\alpha}: A \rightarrow \mathscr{B}\right\}$ such that, if the domain of $f$ is $A$ and $F_{A}^{\alpha}(f)=0$ for all $\alpha$, then $f=0$. Then $J_{s}$ is an embedding. 
Proof. If $J(f)=0$ then by Lemma 1 there is a factoring:

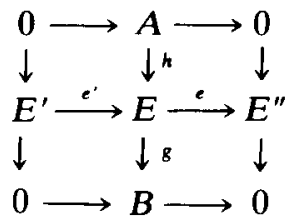

with $f=g h$ and $E^{\prime} \rightarrow E \rightarrow E^{\prime \prime}$ in $\overline{\mathscr{E}}$. Apply $F_{A}^{\alpha}$ to this diagram. Let $K \rightarrow F_{A}^{\alpha}(E)=\operatorname{Ker} F_{A}^{\alpha}(e)$ and let $F_{A}^{\alpha}(E) \rightarrow C=\operatorname{cok} F_{A}^{\alpha}(e)$. Since $F_{A}^{\alpha}(A) \rightarrow F_{A}^{\alpha}(E) \rightarrow F_{A}^{\alpha}\left(E^{\prime \prime}\right)=0$ and $F_{A}^{\alpha}\left(E^{\prime}\right) \rightarrow F_{A}^{\alpha}(E) \rightarrow F_{A}^{\alpha}(B)=0$, there are commutative diagrams:
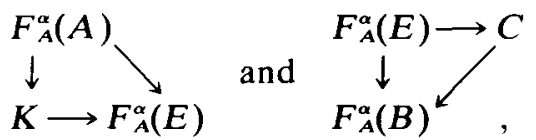

and so:

$$
F_{A}^{\alpha}(A) \rightarrow F_{A}^{\alpha}(B)=F_{A}^{\alpha}(A) \rightarrow K \rightarrow F_{A}^{\alpha}(E) \rightarrow C \rightarrow F_{A}^{\alpha}(B) ;
$$

but $K \rightarrow F_{A}^{\alpha}(E) \rightarrow C=0$, so $F_{A}^{\alpha}(f)=0$, and hence $f=0$.

Definition 2.3. Let $(\mathscr{A}, \mathscr{E})$ be a category with exactness. An object $P$ in $\mathscr{A}$ is called an $\mathscr{E}$-projective provided the sequence

$$
\left(P, E^{\prime}\right) \rightarrow(P, E) \rightarrow\left(P, E^{\prime \prime}\right)
$$

has zero homology for all $E^{\prime} \rightarrow E \rightarrow E^{\prime \prime}$ in $\mathscr{E}$. An $\mathscr{E}$-injective is defined dually.

COROLlaRy 2.4. Let $(\mathscr{A}, \mathscr{E})$ be a category with exactness where $\mathscr{E}$ has the property that for every $A$ in $\mathscr{A}$ there is a jointly epimorphic family $\left\{P_{A}^{\alpha} \rightarrow A\right\}$ with $P_{A}^{\alpha}$ an $\mathscr{E}$-projective for all $\alpha$ and $A$, then $J_{\mathscr{A}}$ is an embedding.

Proof. Let $F_{A}^{\alpha}=\mathscr{A}\left(P_{A}^{\alpha},-\right)$ and let $\mathscr{B}=\mathscr{G}$ then apply theorem 2.2. The dual of Corollary 2.4 is obviously true.

\section{Determination of $\overline{\mathscr{E}}$ by projectives}

Notation 3.1 . Let $(\mathscr{A}, \mathscr{E})$ be an additive category with exactness. Let $\hat{\mathscr{E}}$ defined as:

$$
\begin{gathered}
\hat{\mathscr{E}}=\left\{E^{\prime} \rightarrow E \rightarrow E^{\prime \prime} \mid \mathscr{A}\left(P, E^{\prime}\right) \rightarrow \mathscr{A}(P, E) \rightarrow \mathscr{A}\left(P, E^{\prime \prime}\right)\right. \text { has zero homology for } \\
\text { all } \mathscr{E} \text {-projectives } P\} .
\end{gathered}
$$

It is quite clear that $\overline{\mathscr{E}} \subset \hat{\mathscr{E}}$. If $\mathscr{F}$ is the category of finite abelian groups, there are no projectives and $\hat{E}$ consists of all objects of $\vec{F}$. On the other hand $\overline{\mathscr{E}}$ does not since the inclusion into $\mathscr{G}$ must be exact. 
THEOREM 3.2. Let $(\mathscr{A}, \mathscr{E})$ be an additive category with exactness satisfying the following three conditions:

1. For every $f: E \rightarrow E^{\prime \prime}$ in $\mathscr{A}$ there is a complex $E^{\prime} \rightarrow E \rightarrow E^{\prime \prime}$ in $\mathscr{E}$.

2. If $E \rightarrow E^{\prime \prime}$ is epi then $E \rightarrow E^{\prime \prime} \rightarrow 0$ is in $\mathscr{E}$.

3. For every $A$ in $\mathscr{A}$ there is an epi $P \rightarrow A$ in $\mathscr{A}$ with $P$ an $\mathscr{E}$-projective. Then $\hat{\mathscr{E}}=\overline{\mathscr{E}}$.

Proof. Suppose that $E^{\prime} \rightarrow E \rightarrow E^{\prime \prime}$ is in $\mathscr{E}$, that $\mathscr{B}$ is abelian and that $F$ is an exact functor from $\mathscr{A}$ to $\mathscr{B}$. There is a factoring

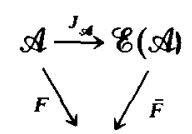

$\mathscr{B}$

with $\vec{F}$ exact (Adelman (1973) 2.1). Hence to show that $F E^{\prime} \rightarrow F E \rightarrow F E^{\prime \prime}$ has zero homology, it suffices to show that $J E^{\prime} \rightarrow J E \rightarrow J E^{\prime \prime}$ does. Let $K \rightarrow E \rightarrow E^{\prime \prime}$ be a complex in $\mathscr{E}$ guaranteed by condition 1 . Then $K \rightarrow E \rightarrow E^{\prime \prime}$ is the cokernel of

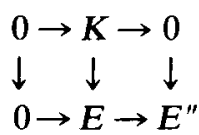

in $\mathscr{E}(\mathscr{A})$. Since $K \rightarrow E \rightarrow E^{\prime \prime}$ is zero in $\mathscr{E}(\mathscr{A})$, the map (1) is epi (Adelman (1973) 1.1). But $0 \rightarrow E \rightarrow E^{\prime \prime}=\operatorname{Ker} J e$ in $A b(\mathscr{A})$ which gives the diagram

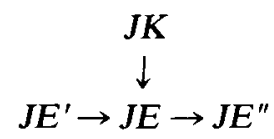

Let $P \rightarrow K$ be the epi with $P$ an $\mathscr{E}$-projective. Since $K \rightarrow E \rightarrow E^{\prime \prime}=0$ it follows that $P \rightarrow K \rightarrow E \rightarrow E^{\prime \prime}=0$; but, since $P$ is $\mathscr{E}$-projective and $E^{\prime} \rightarrow E \rightarrow E^{\prime \prime}$ is in $\mathscr{E}$, there is a map $P \rightarrow E^{\prime}$ so that

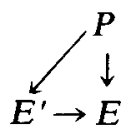

commutes. Apply $J$ to obtain

$$
J P \rightarrow J K \rightarrow \mathrm{Ker} J e \rightarrow J E \rightarrow \mathrm{Cok} J e^{\prime}=J P \rightarrow J E^{\prime} \rightarrow J E \rightarrow \operatorname{Cok} J e^{\prime}=0
$$

Condition 2 guarantees that $J P \rightarrow J K$ is epi in $\mathscr{E}(\mathscr{A})$. Since $J K \rightarrow \operatorname{Ker} J e$ is epi it follows that $\operatorname{Ker} J e \rightarrow J E \rightarrow \operatorname{Cok} J e^{\prime}=0$. But the image of this map is the homology of $J E^{\prime} \rightarrow J E \rightarrow J E^{\prime \prime}$.

The dual of this theorem is also true: 


\section{Examples}

Definttion 4.1. An additive category is called a left Puppe category if every map has a weak kernel and appears as a weak kernel. It is called a right Puppe category if every map has a weak cokernel and appears as a weak cokernel.

Theorem 4.2. Let $A$ be a left (right) Puppe category. Then there is an

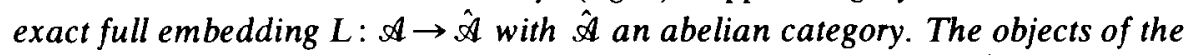
form $L(A)$ form a covering family of projectives (injectives) in $\hat{A}$. Given any weak kernel (cokernel) preserving functor $F: \mathscr{A} \rightarrow \mathscr{B}$ with $\mathscr{B}$ abelian. There is up to natural equivalence a unique exact functor $\hat{F}: \hat{A} \rightarrow \mathscr{B}$ such that

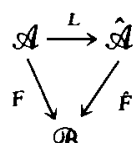

$\mathscr{B}$

commutes. (We will consider only left Puppe categories.)

Proof. Let $\mathscr{E}$ be the class of sequences of the form $E^{\prime} \rightarrow E \rightarrow E^{\prime \prime}$ where $E^{\prime} \rightarrow E$ is a weak kernel of $E \rightarrow E^{\prime \prime} .(\mathscr{A}, \mathscr{E})$ is an additive category with exactness satisfying conditions 1,2 , and 3 of Theorem 3.2. Hence a functor with abelian codomain out of $\mathscr{A}$ will be exact if and only if it preserves weak kernels. Let $\hat{\mathscr{A}}=\mathscr{E}(\mathscr{A})$ and $L=J_{s \&}$. That $L(A)$ are all projectives is found in [1] (2.3.) The fact that $L$ is full follows from Adelman (1973) 2.2. That $L$ is an embedding follows from $\$ 2$.

\section{When the free abelian category is well-powered}

Theorem 3.2 gives conditions on $\mathscr{A}$ and $\mathscr{E}$ that guarantee that $\overline{\mathscr{E}}$ be determined by relative projectives. In many specific cases the class of relative projectives can be assumed to be a set since only one member from each isomorphism class need be used. The next step in generalizing this theorem is to ask: When is $\overline{\mathscr{E}}$ determined by a set of functors into the category of abelian groups? We have no answer to this question but present an answer to the following less general question: What are the conditions on $\mathscr{A}$ so that for all $\mathscr{E}$, the class $\overline{\mathscr{E}}$ will be determined by a set of functors into the category of abelian groups?

Proposition 5.1. If $\overline{\mathscr{E}}$ is determined by a set of functors into $\mathscr{G}$ then it is determined by a single functor into $\mathcal{G}$.

Proof. If $\mathbf{G}$ is the class of functors that determine $\overline{\mathscr{E}}$ let $F=\Pi_{\mathbf{G}} G$. Then $F$ will determine $\overline{\mathscr{E}}$. 
Definition 5.2. If $\overline{\mathscr{E}}$ is determined by a single functor into $\mathscr{G}$ then $(\mathscr{A}, \mathscr{E})$ will be called $\mathscr{G}$-determined.

Definition 5.3. (Freyd (1973) 83). An abelian category is called abelian concrete if there is an exact faithful functor from it to $\mathscr{G}$.

Proposition 5.4. $(\mathscr{A}, \mathscr{E})$ is $\mathscr{G}$-determined if and only if $\mathscr{E}(\mathscr{A})$ is abelian concrete.

Proof. Suppose $(\mathscr{A}, \mathscr{E})$ is $\mathscr{G}$-determined. Let $F: \mathscr{A} \rightarrow \mathscr{G}$ be the determining functor, and let $\hat{F}: \mathscr{E}(\mathscr{A}) \rightarrow \mathscr{G}$ be the unique exact functor such that

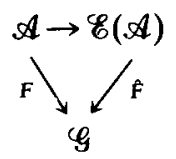

commutes. Then $(E)=0$ iff $(E) \in \overline{\mathscr{C}}$ iff $\hat{F}(\mathscr{E})=0$ hence $\hat{F}$ is faithful. The converse is trivial.

LemMA 5.5. If $\mathscr{A}$ is a well-powered abelian category and $\mathscr{S}$ is a Serre class then $\mathscr{A} / \mathscr{S}$ is a well-powered abelian category.

Proof. Let the diagram

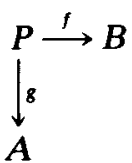

represent the map $g f^{-1}$ in $\mathscr{A} / \mathscr{S}$. Then $g$ is mono in $\mathscr{A} / \mathscr{S}$ iff Ker $g \in \mathscr{S}$. So such a diagram represents a subobject of $\mathscr{A} / \mathscr{S}$. Clearly $g$ and $g f^{-1}$ represent the same subobject so we need consider only subobjects of the form $P \stackrel{B}{\rightarrow} A$ with Ker $g \in \mathscr{P}$ and $g \in \mathscr{A}$. Let $P \stackrel{k}{\longrightarrow} I_{g} \stackrel{\iota}{\longrightarrow} A$ represent the image of $g$. Then $I_{g} \longrightarrow A$ is a subobject of $A$ in $\mathscr{A}$. Given

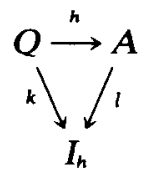

with $\operatorname{Ker} h \in \mathscr{S}$. Suppose $I_{g} \longrightarrow A$ and $I_{g} \longrightarrow A$ represent the same subobject of $A$ in $\mathscr{A}$. Then there is an isomorphism $p: I_{8} \longrightarrow I_{h}$ so that $n p=l$. Now Ker $k=\operatorname{Ker} g \in \mathscr{P}$ and Ker $m=\operatorname{Ker} h \in \mathscr{F}$; and since Cok $h$ and Cok $m$ are both zero and hence in $\mathscr{P}$, it follows that $k$ and $m$ are isomorphisms in $\mathscr{A} / \mathscr{S}$. The diagram 


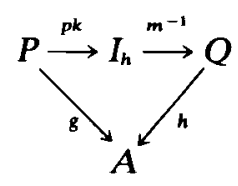

commutes in $\mathscr{A} / \mathscr{S}$ because

$$
h=m^{-1} p k=(n m) m^{-1} p k=n p k=l k=g
$$

So $P \underset{B}{\longrightarrow} A$ and $Q \longrightarrow A$ represent the same subobject of $A$ in $\mathscr{A} / \mathscr{S}$. We have constructed a monomorphism from the subobjects of $A$ in $\mathscr{A} / \mathscr{S}$ into the subobjects of $A$ in $\mathscr{A}$ and so $\mathscr{A} / \mathscr{S}$ is well-powered.

Remark 5.6. The converse is clearly false. For any category $\mathscr{A}$ with pullbacks there is a functor Sub: $\mathscr{A}^{\text {op }} \longrightarrow \mathscr{P}$ ets which assigns to every object the set of subobjects.

LeMma 5.7. Let $A$ be a category with images in which pullbacks of epis are epi. Then the functor Sub preserves monos.

Proof. Suppose $f: P \rightarrow B$ is epi in $\mathscr{A}$. Suppose $A \rightarrow B$ and $C \rightarrow B$ are subobjects of $B$ form the diagram

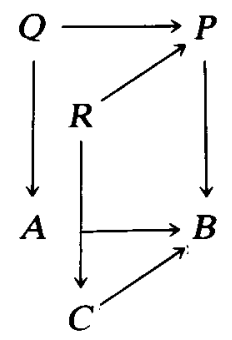

with $Q \rightarrow P$ and $R \rightarrow P$ the images of $A \rightarrow B$ and $C \rightarrow B$ respectively under Sub $(f)$. An isomorphism $Q \rightarrow T$ establishes both $A$ and $C$ as the images of $Q \rightarrow P \rightarrow B$ and so they are the same subobject of $B$.

Definition 5.8. Let $A$ be any category and $A$ an object. Define the following equivalence relation on maps out of $A$ :

$$
f: A \rightarrow B \equiv g: A \rightarrow C \text { if } f \text { factors through } g \text { and } g \text { factors through } f .
$$

The set of equivalence classes of such maps will be called the map class of $A$ denoted by $\mu_{A}$.

TheOREM 5.9. Let $A$ be an additive category. The free abelian category, $\mathrm{Ab}(\mathscr{A})$, on $\mathscr{A}$ is well-powered if and only if $\mathcal{M}_{\mathrm{A}}$ is small for all $A$ in $\mathscr{A}$. 
Proof. Let $(X)$ be in $A b(\mathscr{A})$. There is an epi (Adelman (1973) \$1) with $(K)$ in $K(\mathscr{A})$. Hence $\operatorname{Sub}(X) \rightarrow \operatorname{Sub}(K)$ is mono and so it suffices to show that the objects of $K(\mathscr{A})$ form a small set. Every object of $K(\mathscr{A})$ is a subobject of an object of $\mathscr{A}$ and hence we need only show that $\mathscr{A}$ has a small set of subobjects. Let $(X)$ be a subobject of $A$. Then there is a diagram of the form

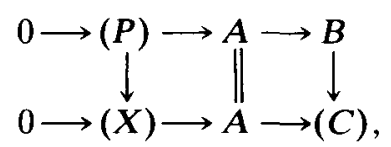

where the left hand square is a pullback. $B \rightarrow(C)$ is an epi with $B$ in $A$ (Adelman (1973) §1). The map $A \rightarrow B$ comes from the fact that $A$ is projective $(C)$ is in $C(A)$ (Adelman (1973) $\$ 1$ ). Both rows are exact. Let $\Re \operatorname{er}(A)$ be the class of all subobjects of $A$ that appear as kernels of maps in $A$. Then the assignment of $(X) \rightarrow A$ to $(P) \rightarrow A$ establishes a monomorphism from $\operatorname{Sub}(A)$ to $\mathscr{K} \operatorname{er}(A)$. It thus suffices to show that $\mathscr{K} \operatorname{er}(A)$ is small. An element of $\mathscr{K} \operatorname{er}(A)$ can be represented as:
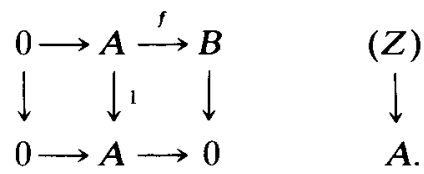

A.

Given another subobject $0 \rightarrow A \rightarrow C=(U)$ containing $(Z)$ there is a diagram
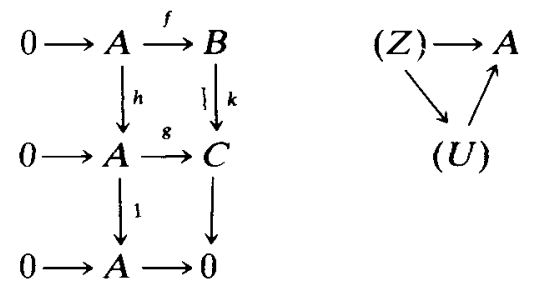

with $s k=1-h$. But the map from $(Z) \rightarrow(U)$ is homotopic to

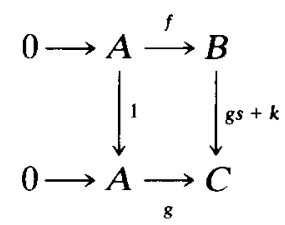

That is, $(Z) \subset(U)$ if and only if $g$ factors through $f$; they are equivalent if and only if $f$ factors through $g$ as well. Hence the class $\mathscr{K}$ er $(A)$ is in bijection with $\mathcal{M}_{A}$ which by hypothesis is small. The converse is now clear because $\mathscr{K} \operatorname{er}(A)$ is bounded by $\operatorname{Sub}(A)$. 
Corollary 5.9. Let $\mathscr{A}$ be an abelian category then $(\mathscr{A}, \mathscr{E})$ will be $\mathscr{G}$-determined for any $\mathscr{E}$ if and only if $\mu_{A}$ is small for all $A$ in $\mathscr{A}$.

Proof. $\quad$ By (5.5), $\mathrm{Ab}(\mathscr{A}) / \mathscr{S}$ will be well-powered for all Serre classes $\mathscr{S}$ if $\mathrm{Ab}(\mathscr{A})$ is well-powered, and so the result follows from (5.8.)

\section{References}

M. Adelman (1973), 'Abelian Categories over Additive Ones', J. of Pure and Applied Algebra 3, 103-117

R. Faber and P. Freyd (1965), 'Fill-in Theorems', Proc. Conf. on Categorical Algebra, La Jolla, (Springer, 1966)

P. Freyd (1965), 'Representations in Abelian Categories', Proc. Conf. in Categorical Algebra, La Jolla, (Springer, 1966)

P. Freyd (1973), 'Concreteness', J. of Pure and Applied Algebra 3, 171-191.

P. Gabriel and M. Zisman, Calculus of Fractions and Homotopy Theory, (Springer, 1967)

Department of Mathematics, Macquarie University, N.S.W. 2113, Australia. 